\title{
RNA Sequencing of Formalin-Fixed, Paraffin-Embedded Specimens for Gene Expression Quantification and Data Mining
}

\author{
Yan Guo, ${ }^{1}$ Jie Wu, ${ }^{2}$ Shilin Zhao, ${ }^{1}$ Fei Ye, ${ }^{3}$ Yinghao Su, ${ }^{2}$ Travis Clark, ${ }^{4}$ Quanhu Sheng, \\ Brian Lehmann, ${ }^{5}$ Xiao-ou Shu, ${ }^{2}$ and Qiuyin Cai ${ }^{2}$ \\ ${ }^{1}$ Department of Cancer Biology, Vanderbilt University, Nashville, TN, USA \\ ${ }^{2}$ Division of Epidemiology, Department of Medicine, Vanderbilt Epidemiology Center and Vanderbilt-Ingram Cancer Center, \\ Vanderbilt University Medical Center, Nashville, TN, USA \\ ${ }^{3}$ Department of Biostatistics, Vanderbilt University, Nashville, TN, USA \\ ${ }^{4}$ Genentech, Baltimore, MD, USA \\ ${ }^{5}$ Department of Biochemistry, Vanderbilt University, Nashville, TN, USA
}

Correspondence should be addressed to Qiuyin Cai; qiuyin.cai@vanderbilt.edu

Received 24 May 2016; Accepted 6 September 2016

Academic Editor: Brian Wigdahl

Copyright (C) 2016 Yan Guo et al. This is an open access article distributed under the Creative Commons Attribution License, which permits unrestricted use, distribution, and reproduction in any medium, provided the original work is properly cited.

Background. Proper rRNA depletion is crucial for the successful utilization of FFPE specimens when studying gene expression. We performed a study to evaluate two major rRNA depletion methods: Ribo-Zero and RNase H. RNAs extracted from 4 samples were treated with the two rRNA depletion methods in duplicate and sequenced $(N=16)$. We evaluated their reducibility, ability to detect RNA, and ability to molecularly subtype these triple negative breast cancer specimens. Results. Both rRNA depletion methods produced consistent data between the technical replicates. We found that the RNase $\mathrm{H}$ method produced higher quality RNAseq data as compared to the Ribo-Zero method. In addition, we evaluated the RNAseq data generated from the FFPE tissue samples for noncoding RNA, including lncRNA, enhancer/super enhancer RNA, and single nucleotide variation (SNV). We found that the $\mathrm{RNase} \mathrm{H}$ is more suitable for detecting high-quality, noncoding RNAs as compared to the Ribo-Zero and provided more consistent molecular subtype identification between replicates. Unfortunately, neither method produced reliable SNV data. Conclusions. In conclusion, for FFPE specimens, the RNase H rRNA depletion method performed better than the Ribo-Zero. Neither method generates data sufficient for SNV detection.

\section{Background}

Formalin-fixed paraffin-embedded (FFPE) tissue is the most common method of tissue preparation used in clinics. FFPE preservation was developed to maintain morphology without any special considerations of preserving nucleic acids. Therefore, the difficulty of evaluating gene expression levels in FFPE samples remains one of the biggest disadvantages of FFPE preservation because the process of fixing the tissue samples and embedding them in paraffin often leads to RNA degradation and chemical modification. Furthermore, a nucleic acid can be cross-linked with a protein during the formalin fixation process, and most of the RNA isolated from FFPE tissues is highly degraded and reduced to a much lower yield than that of RNA isolated from the same amount of fresh tissues. To that end, RNA isolated from recently embedded tissues will be of better quality than RNA isolated from older embedded tissues. As a result, when amplifying RNA with oligo-dT primers, there is an overrepresentation of $3^{\prime}$ data due to the fragmented nature of RNA isolated from FFPE tissues.

Given the aforementioned reasons, gene expression analysis based on FFPE samples has been historically challenging. The most critical step in a FFPE sample based study is tissue preparation, as it ensures the integrity of the yield and data quality. It has been greatly emphasized that improper FFPE tissue preparation can diminish the quality of the nucleic acids from the tissue, limiting their use for gene expression profiling [1]. Yet, FFPE samples are often sought after due to their in-depth retrospective records. The success 
of a FFPE sample based study often depends on several steps: RNA isolation, reverse transcription, qPCR primer design, and preamplification. With carefully designed preparation protocols, FFPE samples have been proven to be an invaluable source for gene expression studies. The potential applications of FFPE samples in biomedical research are substantial.

The vast majority of cellular RNA ( $>80 \%)$ is composed of noninformative ribosomal RNAs (rRNAs, 28 S, 5.8 S, and $18 \mathrm{~S}$ rRNAs) that require removal prior to cDNA synthesis for a RNA-seq library. For high-quality RNA samples, polyadenylated RNA is enriched from intact RNA using oligo-dT primers. Since the rRNA does not have a poly-A tail, it is removed prior to CDNA synthesis along with other informative, non-polyA RNA species. RNA samples isolated from FFPE tissues have two features that are not compatible with oligo-dT primer selection: fragmented RNA that produces $3^{\prime}$ bias from oligo-dT selection, and, the degradation of the poly-A tail, thereby impacting the yield of recovered mRNA. Currently, there are two major rRNA depletion methods used for RNA isolated from FFPE samples: the Ribo-Zero rRNA removal kit (Epicentre/Illumina) and the RNase $\mathrm{H}$ method (also known as SDRNA) [2-4]. The Ribo-Zero kit uses a biotinylated antisense set of DNA capture probes that preferentially bind to rRNA. Magnetic beads are then used to capture the rRNA:DNA capture probe duplex. The resulting non-rRNA is left for cDNA synthesis. The RNase $\mathrm{H}$ method uses a similar initial depletion strategy by annealing 5080 bp antisense DNA probes to the rRNA forming RNA:DNA hybrids. The RNA:DNA hybrids are treated with endoribonuclease RNase $\mathrm{H}$ that specifically degrades the phosphodiester bonds of RNA hybridized to DNA. This step is followed by a DNase I treatment to degrade the excess DNA probes. The resulting RNA is then ready for $\mathrm{CDNA}$ synthesis.

In the 2000s, microarray technology dominated highthroughput gene expression profiling but has since been replaced by RNAseq technology [5-9]. Successful gene expression studies based on FFPE samples by microarray technology [10-12] are much more abundant than studies using the relatively newer RNAseq technology. Here, we apply both RNA depletion methods, Ribo-Zero and RNase $\mathrm{H}$, to isolated RNA from FFPE specimens to compare the overall qualities of data.

Furthermore, based on the premise that sequencing data offers exciting opportunities for additional data mining [13, $14,16]$, we examined the data mining practicability of three types of supplementary information: SNVs, lncRNAs, and enhancer RNAs. SNVs are traditionally identified through DNA samples. SNV detection through RNAseq data has been historically challenging, although, with careful quality control, SNVs are detectable in RNAseq data [17-20]. Long noncoding RNAs (lncRNAs) are arbitrarily defined as longer than 200 nucleotides in length and do not encode proteins. Recent findings have suggested that lncRNAs play important roles in various diseases [21-28], and lncRNAs are detectable through the total RNAseq preparation method by the RiboZero RNA rRNA removal kit [29]. Enhancer RNAs are a type of RNA that regulate spatiotemporal gene expression and impart cell-specific transcriptional outputs [30]. Recent advancements in RNAseq technology have enabled the ready detection of enhancer RNA $[15,30]$. Super enhancer RNAs are a subset of enhancer RNA that are associated with cell identity and genetic risk of various diseases [31-33]. Our unique set of FFPE RNAseq data allows us to answer the question of whether a FFPE sample based RNAseq can be used for these types of data mining and determine which RNA isolation kit produces data most ideal for data mining.

\section{Methods}

2.1. Sample Description. To evaluate the practicability and effectiveness of gene expression profiling using FFPE samples, we designed a study using four triple negative breast cancer (TNBC) FFPE tumor tissue samples. The H\&E slides were reviewed by a study pathologist and tumor tissues were dissected from an unstained FFPE tissue section for total RNA extraction. The tumor tissue sections were stored in a vacuum chamber at $4^{\circ} \mathrm{C}$ for eight to nine years before RNA isolation was performed. Total RNA was extracted and purified using a Qiagen's miRNeasy FFPE Kit, a kit specifically designed for purifying the total RNA and microRNA from FFPE tissue sections. The input RNA amount for both Ribo-Zero and RNase $\mathrm{H}$ rRNA depletion methods was $200 \mathrm{ng}$ each. The quantity and quality of the RNA samples extracted from tumor tissue FFPE sections were checked by Nanodrop (E260, E260/E280 ratio, spectrum 220-320 nm) and by separation on an Agilent BioAnalyzer. Total RNA extracted from each of the four tumors was split into two samples (for a total of eight samples). Two rRNA depletion methods were used: Ribo-Zero and RNase H. Each of the eight samples was treated with the two rRNA depletion methods, prepared for library using TruSeq RNA sample Prep Kit v2 (Illumina), and sequenced by BGI Americas. In total, 16 RNAseq libraries were generated following manufacture protocols and sequenced on two lanes (for a total of eight samples per lane). The qualified libraries were amplified on cBots to generate the cluster on the flow cell. The amplified flow cell was sequenced paired-end on the HiSeq 2000 at read length of the 90 base pairs.

2.2. Data Processing. RNAseq data was thoroughly qualitycontrolled at multiple stages (raw, alignment, and expression) following the recommendation by Guo et al. [34]. Raw data and alignment were quality-controlled using QC3 [35], while expression data was quality-controlled using MultiRankSeq [36]. Alignments were performed using Tophat 2 [37] against the HG19 human reference genome. Read counts for protein coding RNAs, lncRNAs enhancer RNAs, and super enchanter RNAs for each sample were obtained using HTSeq [38] against the collective General Transfer Format (GTF) file build from Ensembl Human GTF v74, Gencode lncRNA v 1.9, and enhancer RNA coordinates provided in [15]. Read count data for each type of the RNA was normalized to the total read counts of each sample. Cluster analysis was performed using Heatmap3 to identify similarities among samples [39]. Spearman's correlation coefficients were used to denote the distance between any two samples. 
TABLE 1: Sample description and alignment statistics.

\begin{tabular}{|c|c|c|c|c|c|c|c|c|}
\hline \multirow{2}{*}{ ID } & \multirow{2}{*}{ Library } & \multicolumn{3}{|c|}{ Raw data } & \multicolumn{4}{|c|}{ Alignment } \\
\hline & & Total reads & BQ & GC & $\mathrm{CR}$ & Non-CR & CR MQ & Non-CR MQ \\
\hline 1 & Ribo-Zero & $17.8 \mathrm{M}$ & 31 & $71.4 \%$ & $27.7 \%$ & $72.3 \%$ & 32 & 47 \\
\hline 2 & Ribo-Zero & $16.1 \mathrm{M}$ & 30 & $76.8 \%$ & $31.4 \%$ & $68.6 \%$ & 23 & 47 \\
\hline 3 & Ribo-Zero & $16.8 \mathrm{M}$ & 31 & $70.7 \%$ & $31.6 \%$ & $68.4 \%$ & 28 & 46 \\
\hline 4 & Ribo-Zero & $14.0 \mathrm{M}$ & 31 & $72.3 \%$ & $46.0 \%$ & $54.0 \%$ & 34 & 47 \\
\hline 1 & Ribo-Zero & $16.1 \mathrm{M}$ & 31 & $70.4 \%$ & $27.4 \%$ & $72.6 \%$ & 31 & 47 \\
\hline 2 & Ribo-Zero & $14.9 \mathrm{M}$ & 31 & $75.4 \%$ & $37.9 \%$ & $62.1 \%$ & 21 & 47 \\
\hline 3 & Ribo-Zero & $17.6 \mathrm{M}$ & 31 & $71.1 \%$ & $29.8 \%$ & $70.2 \%$ & 29 & 47 \\
\hline 4 & Ribo-Zero & $15.6 \mathrm{M}$ & 31 & $70.0 \%$ & $46.1 \%$ & $53.9 \%$ & 36 & 47 \\
\hline 1 & RNase H & $20.2 \mathrm{M}$ & 35 & $51.6 \%$ & $79.9 \%$ & $20.1 \%$ & 46 & 33 \\
\hline 2 & RNase H & $20.5 \mathrm{M}$ & 36 & $39.8 \%$ & $42.6 \%$ & $57.4 \%$ & 45 & 41 \\
\hline 3 & RNase H & $20.4 \mathrm{M}$ & 35 & $51.6 \%$ & $78.9 \%$ & $21.1 \%$ & 45 & 33 \\
\hline 4 & RNase H & $21.4 \mathrm{M}$ & 35 & $48.6 \%$ & $58.0 \%$ & $42.0 \%$ & 45 & 37 \\
\hline 1 & RNase H & $22.1 \mathrm{M}$ & 35 & $52.5 \%$ & $80.3 \%$ & $19.7 \%$ & 46 & 35 \\
\hline 2 & RNase H & $22.4 \mathrm{M}$ & 34 & $55.1 \%$ & $74.7 \%$ & $25.3 \%$ & 44 & 33 \\
\hline 3 & RNase H & $20.6 \mathrm{M}$ & 35 & $52.0 \%$ & $78.5 \%$ & $21.5 \%$ & 45 & 31 \\
\hline 4 & RNase H & $24.0 \mathrm{M}$ & 34 & $53.6 \%$ & $80.1 \%$ & $19.9 \%$ & 45 & 30 \\
\hline
\end{tabular}

CR: coding region; BQ: base quality; MQ: mapping quality; GC: GC content.

2.3. TNBC Subtype. Triple negative breast cancer (TNBC) is known to be molecularly and transcriptionally heterogeneous and can be classified into one of six subtypes (basal-like 1, BL1; basal-like 2, BL2; immunomodulatory, IM; mesenchymal, M; mesenchymal-stem like, MSL; and luminal AR, LAR) based on centroid correlations using gene expression [40]. In order to determine if RNAseq data originated from FFPE specimens can be used for clinical subtyping, we performed TNBC subtyping on each of the samples using TNBCtype [41] and compared the repeatability of TNBC subtyping consistency between the Ribo-Zero and RNase $\mathrm{H}$ methods.

2.4. NanoString. NanoString nCounter data was obtained on 302 genes using the same samples. The detailed processing and normalization method is described in [42]. We computed Spearman's correlation coefficients to evaluate the concordance between RNAseq and NanoString technology.

2.5. SNV Detection. We conducted advanced data mining on our FFPE RNAseq data to extract SNV. We inferred SNVs using Varscan 2 [43]. SNV quality was assessed by the transition/transversion $(\mathrm{Ti} / \mathrm{Tv}$ ) ratio and the pairwise heterozygous genotype consistency rate between any two samples. The $\mathrm{Ti} / \mathrm{Tv}$ ratio is commonly used as a quality control measurement [44-46]. The $\mathrm{Ti} / \mathrm{TV}$ ratio of SNVs residing in coding regions should be between two and three and slightly lower for SNVs residing outside of the coding regions [47]. Higher $\mathrm{Ti} / \mathrm{Tv}$ ratios, without exceeding the upper bound, usually indicate better overall quality. SNVs were annotated with ANNOVAR [48]. The heterozygous consistency rate of a pair of samples A-B is defined as the number of consistent genotypes between samples $A$ and $\mathrm{B}$, divided by the number of total heterozygous genotypes within B. A SNV is qualified as part of a consistency rate computation if it is detected by both samples and if the read depth for that SNV is at least 10 on both samples.

\section{Results}

3.1. Raw Data Quality Assessment. On average, the RiboZero rRNA removal method produced 16.1 (range: 14.017.8) million reads per sample, and the RNase $H$ produced 21.4 (range: 20.2-24.0) million reads per sample. The RNase $\mathrm{H}$ method consistently produced more reads than RiboZero. Given that the same amount of RNA was used and the same number of samples was pooled per lane, a higher RNA capture efficiency is probable for RNase $H$ than that of Ribo-Zero. On average, the guanine-cytosine (GC) content of Ribo-Zero was $72.3 \%$ (range: $70.0-76.8 \%$ ), which was above the expected value (50\%), whereas the GC content of the RNase $\mathrm{H}$ method was 50.6\% (range: 39.8-55.1\%). The GC content of the reference genome is roughly the expected GC content for the sequenced data. The GC content is $39.3 \%$ for the entire human genome, $48.9 \%$ for protein coding RNA, $39.7 \%$ for lncRNA, and $50.2 \%$ for rRNA. The sequenced reads of total RNAseq data are a mixture of protein coding RNA, lncRNA, and other species of RNA. With the expected GC content around 50\%, RNase $\mathrm{H}$ produced data with GC content closest to the expected value. The raw data quality control only provided partial quality assessment of the samples.

3.2. Alignment Quality Assessment. Next, we examined the percentage of the reads that aligned to the coding region (Table 1). For the Ribo-Zero, on average, 34.7\% (range: $27.4-$ $46.1 \%$ ) of the sequenced reads aligned to coding regions, and for the RNase $\mathrm{H}$, on average, $71.6 \%$ (range: $42.6-80.3 \%$ ) of the sequenced reads aligned to coding regions. An interesting 


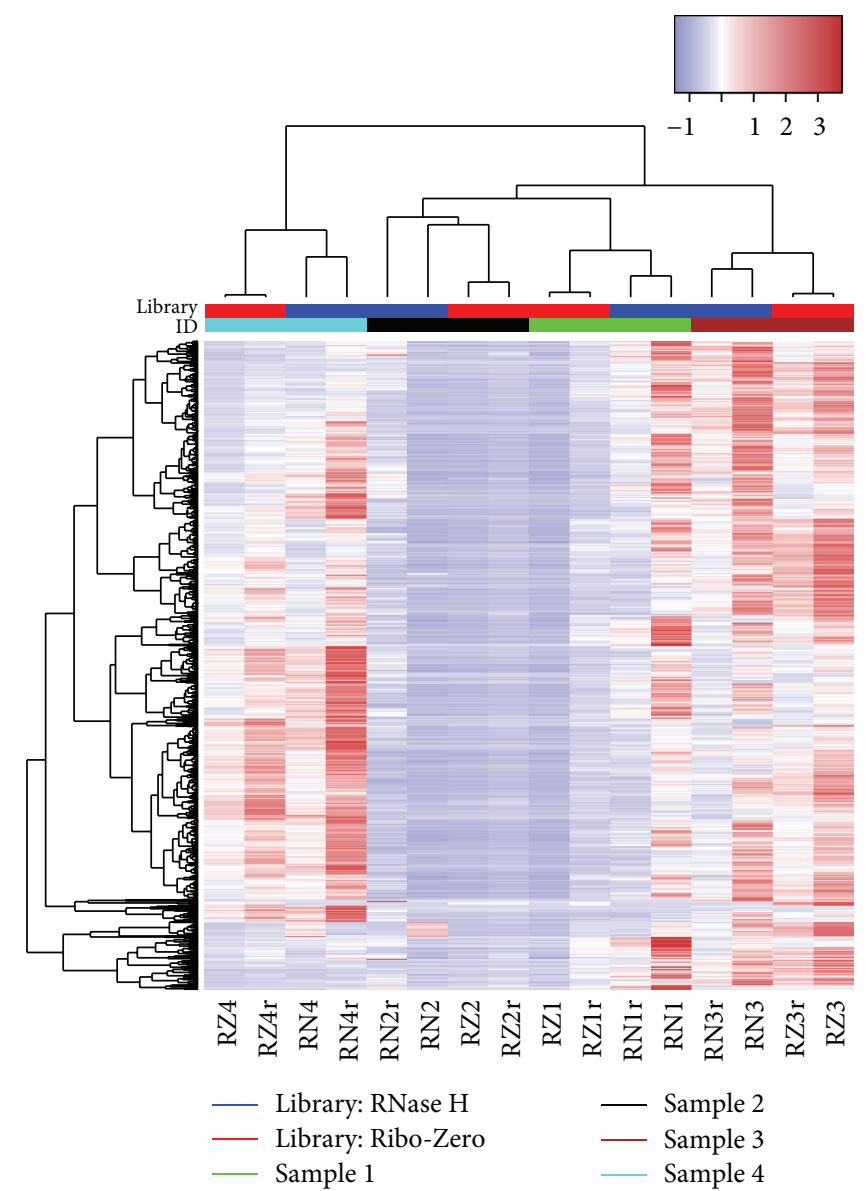

(a)

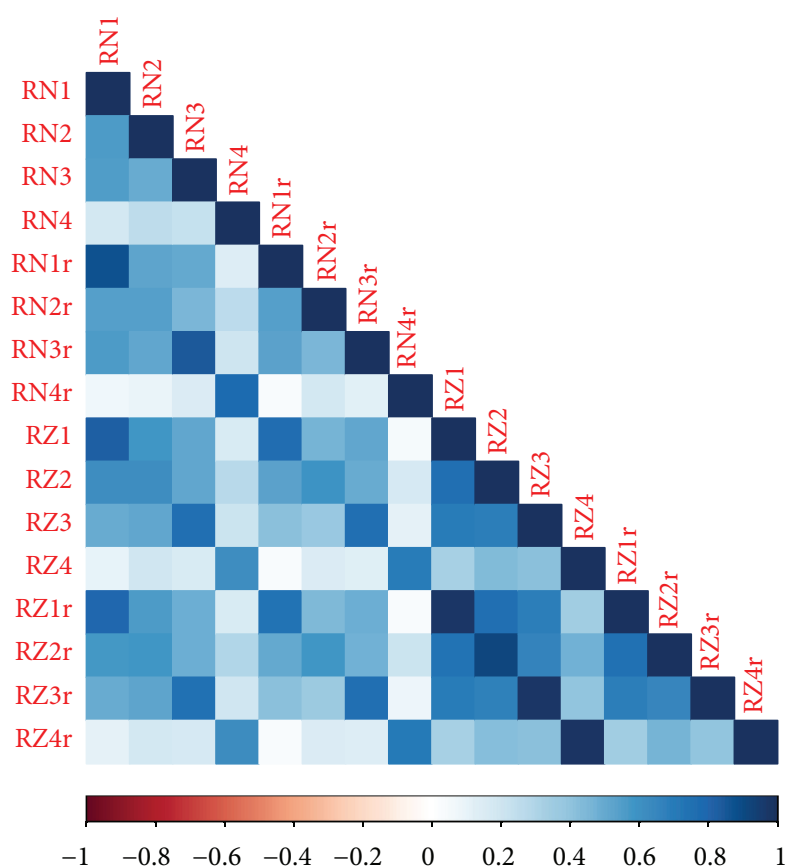

(b)

FIGURE 1: (a) Unsupervised cluster using all detected RNAs. Samples were clustered first by replicates then by rRNA depletion method. (b) Pairwise Spearman correlation heatmap between all samples. Ribo-Zero produced higher correlation between repeats than RNase H. The samples RN4 and RN4r produce low correlations with other samples compared to other random pairs. This could be the result of variation in the sample or variation introduced by the RNase $\mathrm{H}$ kit.

observation was made in regard to the mapping quality (MQ). Ribo-Zero produced higher mapping quality data in the noncoding region, whereas the RNase $\mathrm{H}$ method produced higher mapping quality in the coding region. For the RiboZero, the average MQ for the coding region was 29 (range: 23-36) and 47 (range: 46-47) for the noncoding region. For the RNase $\mathrm{H}$ method, the average MQ was 45 (range: 44-46) for the coding region and 34 (range: $33-41$ ) for the noncoding region. One of the repeats of sample two, which used the RNase $\mathrm{H}$, is a potential outlier because it had the lowest GC content (39.8\%) and the lowest coding region alignment rate (42.6\%) of all the RNase $\mathrm{H}$ based samples. RNase $\mathrm{H}$ also produced less percentage of rRNA reads compared to RiboZero (paired $t$-test $p=0.03$ ).

3.3. Cluster Analysis. Cluster analysis showed that, regardless of which RNA isolation kit was used, the repeated sample clustered together based on gene expression. Within repeated samples, the rRNA depletion kits were clustered separately. The cluster analysis results provided additional evidence of quality concern for the RNase $\mathrm{H}$ sample two repeat one, as it was the only sample that did not perfectly cluster with its pair within the same RNA isolation kit (Figure 1(a)). The correlation heatmap (Figure 1(b)) showed similar results as presented in Figure 1(a). Essentially, we observed a higher pairwise correlation between repeated samples than between random samples.

3.4. TNBC Subtype Comparison. Overall, correlations to the TNBC subtypes were similar in replicates (Figure 2). RNase $\mathrm{H}$ samples had more consistent TNBC subtype calls between replicates (3/4 matching) than the Ribo-Zero samples (2/4 matching). The nonmatching replicate in the RNase H samples is sample 2 where we have previously noted its quality issue. This result suggests that RNase $\mathrm{H}$ produces RNAseq data with more consistent TNBC subtyping.

3.5. NanoString Comparison. We computed Spearman's correlation coefficients using the gene expression levels between RNAseq. The correlation dot plot (Figure 3) shows that the average correlation between Ribo-Zero and NanoString is 0.59 (range: $0.53-0.67$ ), and the average correlation between 


\begin{tabular}{lccccccc}
\hline Sample & BL1 & BL2 & IM & M & MSL & LAR & Subtype \\
\hline RN1 & 0.05 & -0.28 & 0.34 & -0.18 & 0.10 & -0.04 & MSL \\
RN1r & -0.03 & -0.24 & 0.22 & -0.13 & 0.18 & 0.01 & MSL \\
RN2 & -0.30 & 0.00 & 0.00 & -0.08 & 0.24 & 0.25 & LAR \\
RN2r & -0.12 & 0.12 & 0.09 & -0.09 & 0.06 & 0.05 & IM \\
RN3 & 0.34 & -0.19 & -0.18 & 0.23 & -0.10 & -0.11 & LAR \\
RN3r & 0.17 & -0.17 & -0.17 & 0.17 & -0.01 & -0.08 & BL2 \\
RN4 & -0.18 & 0.33 & -0.15 & 0.02 & -0.01 & 0.09 & LAR \\
RN4r & 0.00 & 0.20 & -0.13 & 0.08 & -0.15 & -0.08 & IM \\
RZ1 & -0.12 & -0.17 & 0.08 & -0.12 & 0.16 & 0.20 & BL1 \\
RZ1r & -0.18 & -0.32 & 0.38 & -0.32 & 0.25 & 0.16 & BL1 \\
RZ2 & -0.15 & 0.10 & 0.14 & -0.20 & 0.09 & 0.17 & BL1 \\
RZ2r & -0.21 & 0.22 & 0.26 & -0.29 & 0.06 & 0.12 & BL1 \\
RZ3 & 0.25 & -0.09 & -0.16 & 0.14 & -0.13 & -0.14 & BL2 \\
RZ3r & 0.24 & -0.04 & -0.12 & 0.14 & -0.10 & -0.12 & BL2 \\
RZ4 & -0.12 & 0.29 & -0.12 & 0.02 & -0.15 & 0.01 & BL2 \\
RZ4r & -0.08 & 0.32 & -0.08 & 0.02 & -0.15 & -0.05 & BL2 \\
\hline & & & & & & &
\end{tabular}

BL1: basal-like 1

BL2: basal-like 2

IM: immunomodulatory
M: mesenchymal

MSL: mesenchymal-stem like

LAR: luminal AR

FIgURE 2: TNBC subtype results from TNBC type. The results show that RNase H samples produced better TNBC subtype consistency than Ribo-Zero samples.

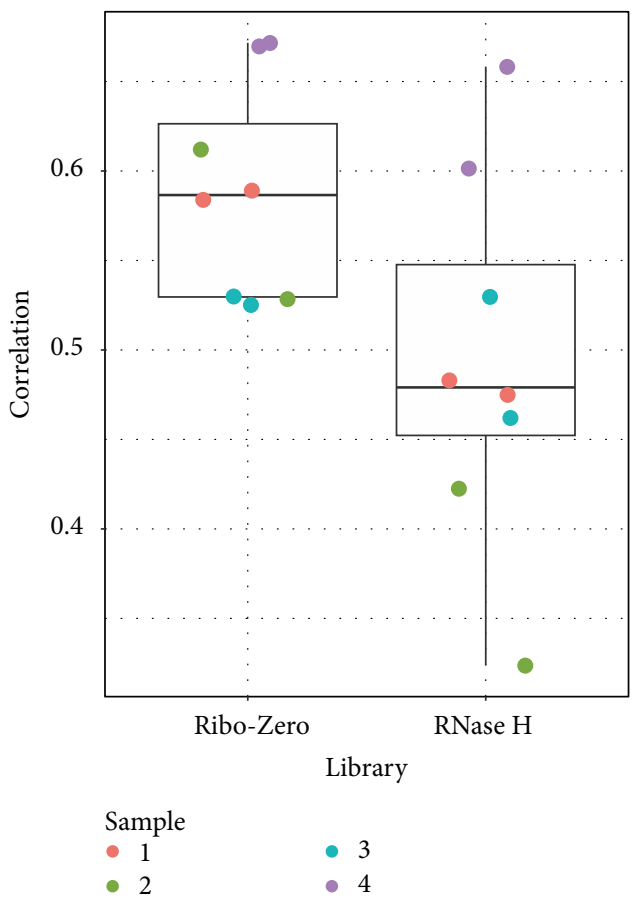

FIGURE 3: Spearman's correlation coefficients between RNAseq data and NanoString data. The Ribo-Zero samples produced slightly higher correlation with NanoString data then RNase H samples.

RNase $\mathrm{H}$ and NanoString is 0.49 (range: 0.32-0.66). The lowest correlation was produced by RNase $\mathrm{H}$ sample two repeat one which is likely to be a sample with a sequencing quality issue.

3.6. RNA Detection. We examined four kinds of RNAs: mRNA (Figure 4(a)), lncRNA (Figure 4(b)), enhancer RNA (Figure 4(c)), and super enhancer RNA (Figure 4(d)). After normalization by total read count, we used four detection thresholds $(>0,>2,>5$, and $>10)$ to compare the RNA detection rates between the two RNA isolation kits. For all four types of RNAs, the Ribo-Zero rRNA depletion method detects more RNA at detection thresholds $>0$ and $>2$. When higher detection thresholds were used, the RNase $\mathrm{H}$ managed to detect more RNAs. RNA detected with low expression values could be the result of noises and is therefore less trustworthy than RNA detected with higher levels of expression. Based on these results, the RNase H rRNA depletion method detected more potentially reliable RNA as compared to the Ribo-Zero.

3.7. SNV Detection. We inferred SNVs from the FFPE RNA data using VarScan 2. After filtering for high quality SNVs (depth > 20), on average, the Ribo-Zero samples identified 525 SNVs per sample (range: 73-1862), and the RNase $\mathrm{H}$ samples identified 57747 SNVs per sample (range: 2193287146). The RNase $H$ samples clearly identified more SNVs than the Ribo-Zero prepared samples. This is caused by the difference of number of callable sites between the two kits. We defined a callable site to be a genomic position with coverage depth $\geq 20$. RNase $H$ produced substantially more 

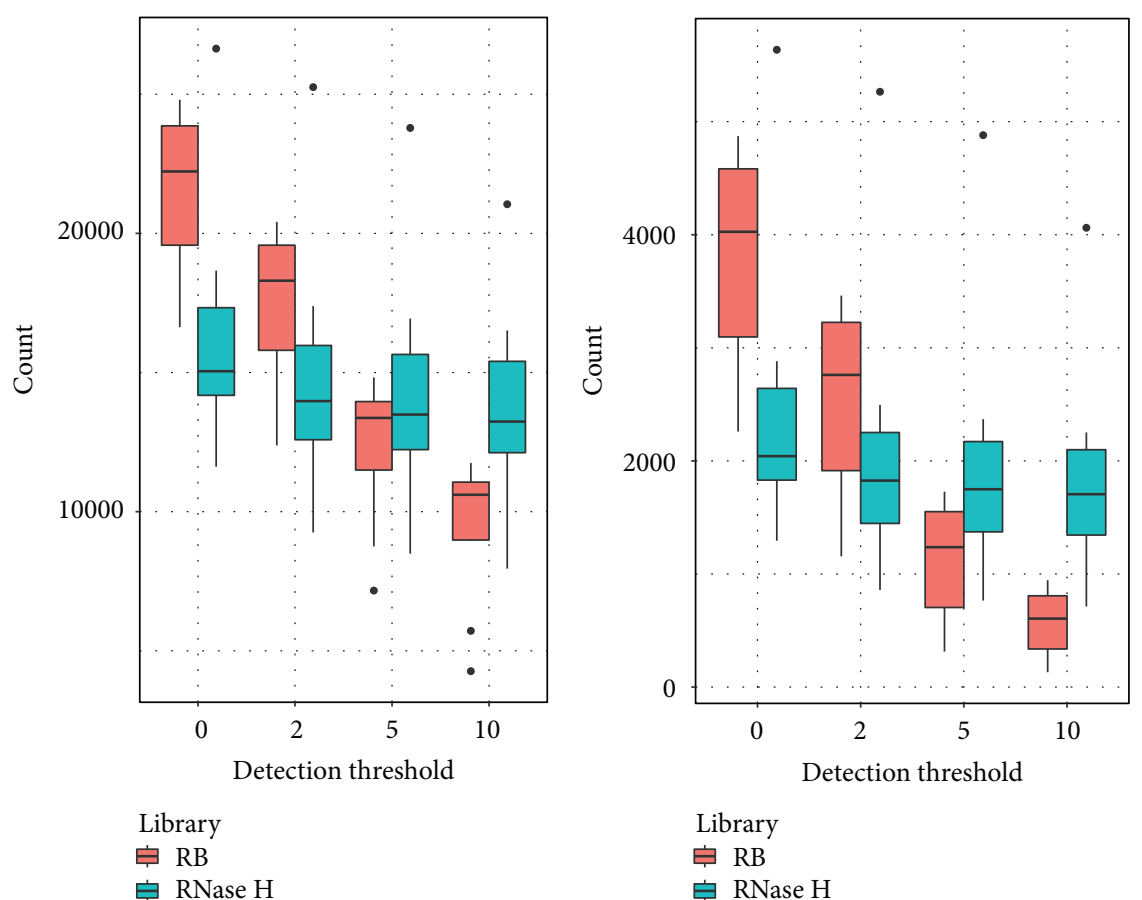

(a)
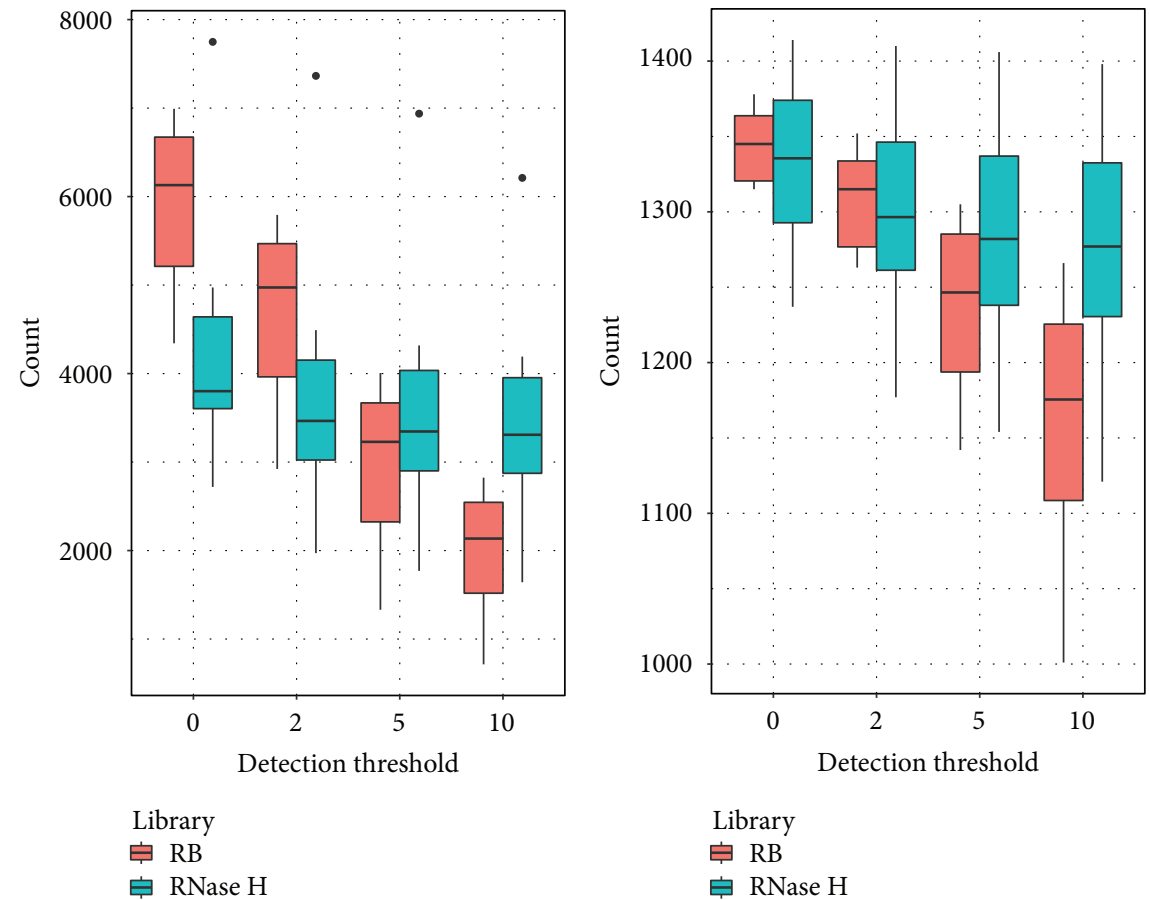

(c)

(d)

FIgURE 4: Detected RNA using thresholds: normalized reads count > 0, 2, 5, and 10. (a) Protein coding RNA. (b) lncRNA. (c) Enhancer RNA. (d) Super enhancer RNA. At lower thresholds (more noise), Ribo-Zero samples detected more RNAs. At higher thresholds (more reliability), RNase $\mathrm{H}$ method detected more RNAs.

callable sites than Ribo-Zero (Figure 5). The callable site analysis result shows that the coverage of Ribo-Zero is more spread out than RNase $\mathrm{H}$. High variations in the number of SNVs were observed for both RNA isolation kits. For
SNVs identified in coding regions, on average, the $\mathrm{Ti} / \mathrm{Tv}$ ratio for Ribo-Zero was 3.51 (range: 2.42-8.00) and 2.08 (range: 1.34-2.47) for RNase H. For SNVs identified in noncoding regions, on average, the $\mathrm{Ti} / \mathrm{Tv}$ ratio for Ribo-Zero was 2.84 


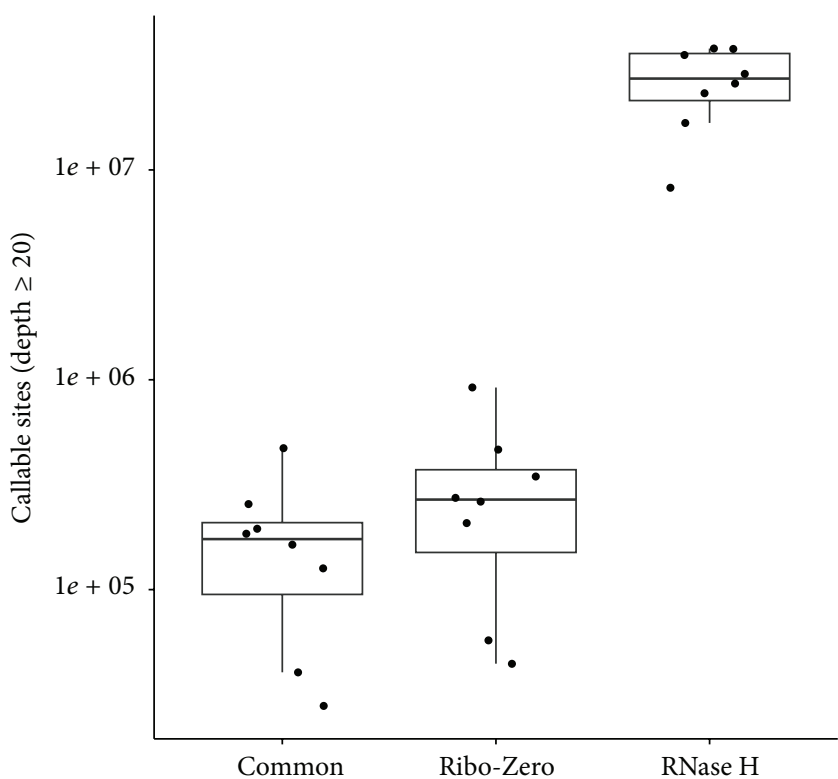

FIGURE 5: Callable site is defined as a genomic position with depth coverage $\geq 20$. The number of callable sites indicates the number of genomic positions that are suitable for SNV inference. RNase $\mathrm{H}$ had substantially more callable sites than Ribo-Zero. The percentage of difference in callable site is significantly more than the percentage of difference in number of total reads sequenced by the two kits. $Y$-axis is plotted in $\log _{10}$ scale.

(range: 2.37-3.84) and 3.74 (range: 1.04-5.27) for RNase $H$. The variation for the $\mathrm{Ti} / \mathrm{Tv}$ ratio is large, indicating potential problems with the SNVs identified.

Additional evidence for problematic SNV inferences was observed in the results of the pairwise heterozygous genotype consistency between samples. In DNA sequencing, we expect the heterozygous genotype consistency rate for technical replicates to be above 0.99 . For RNAseq, the consistency rate is expected to be lower but still yield above $80 \%$. However, on average, the consistency rates for both kits were less than $40 \%$ which were substantially below expectation. Restricting SNV pairwise heterozygous consistency computation to SNVs with depth greater than $50 \mathrm{x}$ for both samples in the pair increased the consistency slightly but still remained $<50 \%$. The low heterozygous consistency rates indicate that SNVs inferred from FFPE RNAseq samples contain high false positive rates and are therefore not ideal sources for detecting SNV.

\section{Discussion}

Utilization of FFPE specimens for gene expression studies could open a new avenue for molecular epidemiological and clinical research. Yet to date, the low quality of RNA from FFPE specimens for gene expression analysis has been a challenge. Several technologies have been developed for quantifying gene expression from FFPE specimens, such as NanoString [49] and quantitative Nuclease Protection Assay [50].
Since gene expression data can yield both molecular subtype classification and predicative markers of risk, efforts have been made to use RNA extracted from FFPE tissue on NanoString and microarray platforms $[51,52]$. Triple negative breast cancer has been shown to be transcriptionally heterogeneous, with several molecular subtypes with differing biology $[40,53,54]$. The ability to identify TNBC subtypes from RNA isolated from FFPE tissues will provide opportunities for future clinical trial designs and retrospective evaluations of previously failed clinical trials by individual subtypes. To determine if RNA extracted from FFPE tissue that has been stored for eight to nine years could yield gene expression profiles by RNAseq sufficient enough to subtype TNBC, we compared the efficiencies of both the Ribo-Zero and the RNase $\mathrm{H}$ methods for rRNA depletion.

Through thorough quality control and analyses, we found that expression profiling of coding and noncoding RNA is possible for aged FFPE samples with RNAseq technology. The Ribo-Zero and RNase $\mathrm{H}$ method each had strengths and weaknesses in different areas. Our analyses suggested that RNase $\mathrm{H}$ is more suitable for studies that target protein coding RNA. On the other hand, Ribo-Zero offered more consistency between repeated samples, which is of pivotal importance, especially for low quality RNA extracted from FFPE tissues. Under the same amount of library input and same multiplexing scenario, RNase $\mathrm{H}$ consistently produced more reads than Ribo-Zero. Many reasons could have caused this read counts difference, including batch effect of the cluster on the flow cell, and library efficiencies. The evidences of more total reads sequenced under the same input amount and better rRNA depletion efficiency for RNase $\mathrm{H}$ support that RNase $\mathrm{H}$ has better library efficiency than Ribo-Zero. RNase $\mathrm{H}$ hybridizes directly to the sequences of rRNAs without the requirement of perfect match. The Ribo-Zero uses bait strategy which is similar to enrichment like exome capture with baits and beads. Thus it does not remove degraded, fragmented rRNAs as efficient as RNase H. Our study confirms previous finding that RNase $\mathrm{H}$ performed better than Ribo-Zero for low quality RNAs [55].

Furthermore, genes quantified from Ribo-Zero processed RNAseq data also had a slightly higher correlation with genes quantified by NanoString technology. This suggests that Ribo-Zero might offer better repeatability, although the correlation (50-60\%, FFPE) with NanoString data (FFPE) did not reach the high correlation (80-90\%, fresh frozen) between microarray and RNAseq [56]. We suspect this is primarily due to the variation introduced by the degraded quality of the RNA extracted from FFPE samples.

The subtyping of gene expression profiles obtained by both methods demonstrated that RNA isolated from stored FFPE samples can be used to determine distinct TNBC subtypes. While TNBC subtypes were similar among replicates, RNase $\mathrm{H}$ samples had more consistent TNBC subtype calls between replicates than that of the Ribo-Zero samples, which is potentially due to the more efficient capture of protein coding RNA.

By performing SNV detection analysis, we found that SNV detected by FFPE RNAseq data is subjected to quality concerns. It has been suggested that the SNV data inferred 
from RNAseq data has a high false positive rate [57]. Several factors can contribute to the high false positive rate of SNV. First, alignment on RNAseq data can be more complicated than DNA sequencing data [19]. Processes such as RNA editing, alternative splicing, gene fusion, and polyadenylation introduce additional complications in RNAseq alignment. The step that reverse-transcribes RNA to cDNA can also introduce random errors. We have found that the number of SNVs inferred from RNAseq data can be several folds higher than that from the exome sequencing data on the sample. In our study, the lower quality of RNA isolated from FFPE tissue will result in an even higher number of false positive SNVs. The low consistency rate of SNVs identified between paired samples suggests that RNAseq data from FFPE tissues are not suitable for SNV inference.

\section{Conclusion}

Recent studies have shown remarkably high consistency between RNAseq data generated from paired freshly frozen and FFPE tissue samples [58-60]. Our study provides additional evidence for the practicability of conducting gene expression RNAseq with FFPE tissues. There is no denying that there are technical and quality limitations for FFPE RNAseq data. However, the majority of the issues can be overcome through thorough quality control and careful bioinformatics analyses. Our study supports the notions that RNAseq on FFPE samples can be used as an unbiased and comprehensive assessment of gene expression in biomedical studies, and RNase $\mathrm{H}$ method provides more efficient rRNA depletion than Ribo-Zero method for low quality fragmented RNAs.

\section{Abbreviations}

FFPE: $\quad$ Formalin-fixed paraffin-embedded

RNAseq: RNA sequencing

SNV: $\quad$ Single nucleotide variant

TNBC: Triple negative breast cancer.

\section{Additional Points}

Availability of Data and Materials. The sequencing data used in this study have been deposited into Gene Expression Omnibus (GEO) under the accession number GSE74270.

\section{Ethical Approval}

The study was approved by the institutional review board of Vanderbilt University.

\section{Consent}

All participants provided written informed consent during in-person interviews.

\section{Competing Interests}

The authors declare that they have no competing interests.

\section{Authors' Contributions}

Yan Guo performed the sequencing analysis and wrote the manuscript. Shilin Zhao assisted with sequencing data analysis. Fei Ye performed the Nanostring data analysis. Jie $\mathrm{Wu}$ prepared the samples. Yinghao $\mathrm{Su}$ prepared the samples. Qiuyin Cai designed the study and contributed to the writing of the manuscript. Brian Lehmann performed the TNBC subtype analysis. Quanhu Sheng performed the TNBC subtype analysis and assisted with sequencing analysis. Travis Clark contributed to writing of the manuscript. Xiao-ou Shu designed the overall study.

\section{Acknowledgments}

Yan Guo was supported by P30 CA068485. We would also like to thank Stephanie Page Hoskins for editorial support. RNAseq and sample collection were supported by R01CA064277, R01CA118229, U01CA161045, and P50CA098131. RNA sample preparation was conducted at the Survey and Biospecimen Shared Resources, which is supported in part by the Vanderbilt-Ingram Cancer Center (P30CA068485).

\section{References}

[1] S. M. Hewitt, F. A. Lewis, Y. Cao et al., "Tissue handling and specimen preparation in surgical pathology: issues concerning the recovery of nucleic acids from formalin-fixed, paraffin-embedded tissue," Archives of Pathology and Laboratory Medicine, vol. 132, no. 12, pp. 1929-1935, 2008.

[2] X. Adiconis, D. Borges-Rivera, R. Satija et al., "Comparative analysis of RNA sequencing methods for degraded or low-input samples," Nature Methods, vol. 10, no. 7, pp. 623-629, 2014, Nature Methods, vol. 11, pp. 210, 2013.

[3] R. Huang, M. Jaritz, P. Guenzl et al., "An RNA-seq strategy to detect the complete coding and non-coding transcriptome including full-length imprinted macro ncrnas," PLOS ONE, vol. 6, no. 11, Article ID e27288, 2011.

[4] J. D. Morlan, K. Qu, and D. V. Sinicropi, "Selective depletion of rRNA enables whole transcriptome profiling of archival fixed tissue," PLoS ONE, vol. 7, no. 8, Article ID e42882, 2012.

[5] Y. W. Asmann, E. W. Klee, E. A. Thompson et al., “3” tag digital gene expression profiling of human brain and universal reference RNA using Illumina Genome Analyzer," BMC Genomics, vol. 10, article 531, 2009.

[6] N. Cloonan, A. R. R. Forrest, G. Kolle et al., "Stem cell transcriptome profiling via massive-scale mRNA sequencing," Nature Methods, vol. 5, no. 7, pp. 613-619, 2008.

[7] Y. Guo, Q. Sheng, J. Li, F. Ye, D. C. Samuels, and Y. Shyr, "Large scale comparison of gene expression levels by microarrays and RNAseq using TCGA data," PLoS ONE, vol. 8, no. 8, Article ID e71462, 2013.

[8] J. C. Marioni, C. E. Mason, S. M. Mane, M. Stephens, and Y. Gilad, "RNA-seq: an assessment of technical reproducibility and comparison with gene expression arrays," Genome Research, vol. 18, no. 9, pp. 1509-1517, 2008.

[9] Z. Wang, M. Gerstein, and M. Snyder, "RNA-Seq: a revolutionary tool for transcriptomics," Nature Reviews Genetics, vol. 10, no. 1, pp. 57-63, 2009. 
[10] X.-J. Ma, Z. Wang, P. D. Ryan et al., "A two-gene expression ratio predicts clinical outcome in breast cancer patients treated with tamoxifen," Cancer Cell, vol. 5, no. 6, pp. 607-616, 2004.

[11] L. Mittempergher, J. J. de Ronde, M. Nieuwland et al., "Gene expression profiles from formalin fixed paraffin embedded breast cancer tissue are largely comparable to fresh frozen matched tissue," PLoS ONE, vol. 6, no. 2, article e17163, 2011.

[12] P. T. Nelson, D. A. Baldwin, L. M. Scearce, J. C. Oberholtzer, J. W. Tobias, and Z. Mourelatos, "Microarray-based, highthroughput gene expression profiling of microRNAs," Nature Methods, vol. 1, no. 2, pp. 155-161, 2004.

[13] L. Han, K. C. Vickers, D. C. Samuels, and Y. Guo, "Alternative applications for distinct RNA sequencing strategies," Briefings in Bioinformatics, vol. 16, no. 4, pp. 629-639, 2014.

[14] D. C. Samuels, L. Han, J. Li et al., "Finding the lost treasures in exome sequencing data," Trends in Genetics, vol. 29, no. 10, pp. 593-599, 2013.

[15] G. Vahedi, Y. Kanno, Y. Furumoto et al., "Super-enhancers delineate disease-associated regulatory nodes in T cells," Nature, vol. 520, no. 7548, pp. 558-562, 2015.

[16] K. C. Vickers, L. A. Roteta, H. Hucheson-Dilks, L. Han, and Y. Guo, "Mining diverse small RNA species in the deep transcriptome," Trends in Biochemical Sciences, vol. 40, no. 1, pp. 4-7, 2015.

[17] I. Chepelev, G. Wei, Q. Tang, and K. Zhao, "Detection of single nucleotide variations in expressed exons of the human genome using RNA-Seq," Nucleic Acids Research, vol. 37, no. 16, article e106, 2009.

[18] A. C. Miller, N. D. Obholzer, A. N. Shah, S. G. Megason, and C. B. Moens, "RNA-seq-based mapping and candidate identification of mutations from forward genetic screens," Genome Research, vol. 23, no. 4, pp. 679-686, 2013.

[19] R. Piskol, G. Ramaswami, and J. B. Li, "Reliable identification of genomic variants from RNA-seq data," American Journal of Human Genetics, vol. 93, no. 4, pp. 641-651, 2013.

[20] E. M. Quinn, P. Cormican, E. M. Kenny et al., "Development of strategies for SNP detection in RNA-Seq data: application to lymphoblastoid cell lines and evaluation using 1000 genomes data," PLoS ONE, vol. 8, no. 3, article e58815, 2013.

[21] P. P. Amaral and J. S. Mattick, "Noncoding RNA in development," Mammalian Genome, vol. 19, no. 7-8, pp. 454-492, 2008.

[22] A. Bhan, I. Hussain, K. I. Ansari, S. A. M. Bobzean, L. I. Perrotti, and S. S. Mandal, "Bisphenol-A and diethylstilbestrol exposure induces the expression of breast cancer associated long noncoding RNA HOTAIR in vitro and in vivo," Journal of Steroid Biochemistry and Molecular Biology, vol. 141, pp. 160170, 2014.

[23] A. Bhan, I. Hussain, K. I. Ansari, S. Kasiri, A. Bashyal, and S. S. Mandal, "Antisense transcript long noncoding RNA (lncRNA) HOTAIR is transcriptionally induced by estradiol," Journal of Molecular Biology, vol. 425, no. 19, pp. 3707-3722, 2013.

[24] M. E. Dinger, P. P. Amaral, T. R. Mercer, and J. S. Mattick, "Pervasive transcription of the eukaryotic genome: functional indices and conceptual implications," Briefings in Functional Genomics and Proteomics, vol. 8, no. 6, Article ID elp038, pp. 407-423, 2009.

[25] M. E. Dinger, P. P. Amara, T. R. Mercer et al., "Long noncoding RNAs in mouse embryonic stem cell pluripotency and differentiation," Genome Research, vol. 18, no. 9, pp. 1433-1445, 2008.

[26] T. R. Mercer, M. E. Dinger, and J. S. Mattick, "Long non-coding RNAs: insights into functions," Nature Reviews Genetics, vol. 10, no. 3, pp. 155-159, 2009.
[27] T. R. Mercer, M. E. Dinger, S. M. Sunkin, M. F. Mehler, and J. S. Mattick, "Specific expression of long noncoding RNAs in the mouse brain," Proceedings of the National Academy of Sciences of the United States of America, vol. 105, no. 2, pp. 716-721, 2008.

[28] S. Schoeftner and M. A. Blasco, "Developmentally regulated transcription of mammalian telomeres by DNA-dependent RNA polymerase II," Nature Cell Biology, vol. 10, no. 2, pp. 228236, 2008.

[29] Y. Guo, S. Zhao, Q. Sheng et al., "RNAseq by total RNA library identifies additional RNAs compared to poly(A) RNA library," BioMed Research International, vol. 2015, Article ID 862130, 9 pages, 2015.

[30] R. Andersson, C. Gebhard, I. Miguel-Escalada et al., "An atlas of active enhancers across human cell types and tissues," Nature, vol. 507, no. 7493, pp. 455-461, 2014.

[31] D. Hnisz, B. J. Abraham, T. I. Lee et al., "Super-enhancers in the control of cell identity and disease," Cell, vol. 155, no. 4, pp. 934947, 2013.

[32] J. Lovén, H. A. Hoke, C. Y. Lin et al., "Selective inhibition of tumor oncogenes by disruption of super-enhancers," Cell, vol. 153 , no. 2, pp. 320-334, 2013.

[33] W. A. Whyte, D. A. Orlando, D. Hnisz et al., "Master transcription factors and mediator establish super-enhancers at key cell identity genes," Cell, vol. 153, no. 2, pp. 307-319, 2013.

[34] Y. Guo, F. Ye, Q. Sheng, T. Clark, and D. C. Samuels, “Three-stage quality control strategies for DNA re-sequencing data," Briefings in Bioinformatics, vol. 15, no. 6, Article ID bbt069, pp. 879-889, 2013.

[35] Y. Guo, S. Zhao, Q. Sheng et al., "Multi-perspective quality control of Illumina exome sequencing data using QC3," Genomics, vol. 103, no. 5-6, pp. 323-328, 2014.

[36] Y. Guo, S. Zhao, F. Ye, Q. Sheng, and Y. Shyr, "MultiRankSeq: multiperspective approach for RNAseq differential expression analysis and quality control," BioMed Research International, vol. 2014, Article ID 248090, 8 pages, 2014.

[37] D. Kim, G. Pertea, C. Trapnell, H. Pimentel, R. Kelley, and S. L. Salzberg, "TopHat2: accurate alignment of transcriptomes in the presence of insertions, deletions and gene fusions," Genome Biology, vol. 14, no. 4, article R36, 2013.

[38] S. Anders, P. T. Pyl, and W. Huber, "HTSeq-A Python framework to work with high-throughput sequencing data," Bioinformatics, vol. 31, no. 2, pp. 166-169, 2015.

[39] S. Zhao, Y. Guo, Q. Sheng, and Y. Shyr, "Advanced heat map and clustering analysis using heatmap3," BioMed Research International, vol. 2014, Article ID 986048, 6 pages, 2014.

[40] B. D. Lehmann, J. A. Bauer, X. Chen et al., "Identification of human triple-negative breast cancer subtypes and preclinical models for selection of targeted therapies," The Journal of Clinical Investigation, vol. 121, no. 7, pp. 2750-2767, 2011.

[41] X. Chen, J. Li, W. H. Gray et al., "TNBCtype: a subtyping tool for triple-negative breast cancer," Cancer Informatics, vol. 11, pp. 147-156, 2012.

[42] M. L. Baglia, Q. Cai, Y. Zheng et al., "Dual specificity phosphatase 4 gene expression in association with triple-negative breast cancer outcome," Breast Cancer Research and Treatment, vol. 148, no. 1, pp. 211-220, 2014.

[43] D. C. Koboldt, Q. Zhang, D. E. Larson et al., "VarScan 2: somatic mutation and copy number alteration discovery in cancer by exome sequencing," Genome Research, vol. 22, no. 3, pp. 568576, 2012. 
[44] R. M. Durbin, D. L. Altshuler, G. R. Abecasis et al., "A map of human genome variation from population-scale sequencing," Nature, vol. 467, no. 7319, pp. 1061-1073, 2010.

[45] Y. Guo, J. Li, C.-I. Li, J. Long, D. C. Samuels, and Y. Shyr, “The effect of strand bias in Illumina short-read sequencing data," BMC Genomics, vol. 13, article 666, 2012.

[46] Y. Guo, J. Long, J. He et al., "Exome sequencing generates high quality data in non-target regions," BMC Genomics, vol. 13, no. 1, article 194, 2012.

[47] J. Wang, L. Raskin, D. C. Samuels, Y. Shyr, and Y. Guo, “Genome measures used for quality control are dependent on gene function and ancestry," Bioinformatics, vol. 31, no. 3, pp. 318323, 2015.

[48] K. Wang, M. Li, and H. Hakonarson, "ANNOVAR: functional annotation of genetic variants from high-throughput sequencing data," Nucleic Acids Research, vol. 38, no. 16, article e164, 2010.

[49] P. P. Reis, L. Waldron, R. S. Goswami et al., "MRNA transcript quantification in archival samples using multiplexed, colorcoded probes," BMC Biotechnology, vol. 11, article 46, 2011.

[50] R. A. Roberts, C. M. Sabalos, M. L. LeBlanc et al., "Quantitative nuclease protection assay in paraffin-embedded tissue replicates prognostic microarray gene expression in diffuse large-B-cell lymphoma," Laboratory Investigation, vol. 87, no. 10, pp. 979997, 2007.

[51] T. Nielsen, B. Wallden, C. Schaper et al., "Analytical validation of the PAM50-based Prosigna Breast Cancer Prognostic Gene Signature Assay and nCounter Analysis System using formalinfixed paraffin-embedded breast tumor specimens," BMC Cancer, vol. 14, article 177, 2014.

[52] A. Sapino, P. Roepman, S. C. Linn et al., "MammaPrint molecular diagnostics on formalin-fixed, paraffin-embedded tissue," Journal of Molecular Diagnostics, vol. 16, no. 2, pp. 190-197, 2014.

[53] M. D. Burstein, A. Tsimelzon, G. M. Poage et al., "Comprehensive genomic analysis identifies novel subtypes and targets of triple-negative breast cancer," Clinical Cancer Research, vol. 21, no. 7, pp. 1688-1698, 2015.

[54] P. Jézéquel, D. Loussouarn, C. Guérin-Charbonnel et al., “Geneexpression molecular subtyping of triple-negative breast cancer tumours: importance of immune response," Breast Cancer Research, vol. 17, article 43, 2015.

[55] X. Adiconis, D. Borges-Rivera, R. Satija et al., "Comparative analysis of RNA sequencing methods for degraded or low-input samples," Nature Methods, vol. 10, no. 7, pp. 623-629, 2013.

[56] Y. Guo, Q. H. Sheng, J. Li, F. Ye, D. C. Samuels, and Y. Shyr, "Large scale comparison of gene expression levels by microarrays and RNAseq using TCGA data," PLoS ONE, vol. 8, no. 8, Article ID e71462, 2013.

[57] Q. Sheng, S. Zhao, C. Li, Y. Shyr, and Y. Guo, "Practicability of detecting somatic point mutation from RNA high throughput sequencing data," Genomics, vol. 107, no. 5, pp. 163-169, 2016.

[58] J. Hedegaard, K. Thorsen, M. K. Lund et al., "Next-generation sequencing of RNA and DNA isolated from paired fresh-frozen and formalin-fixed paraffin-embedded samples of human cancer and normal tissue," PLOS ONE, vol. 9, no. 5, Article ID e98187, 2014.

[59] P. Li, A. Conley, H. Zhang, and H. L. Kim, "Whole-transcriptome profiling of formalin-fixed, paraffin-embedded renal cell carcinoma by RNA-seq," BMC Genomics, vol. 15, article 1087, 2014.
[60] W. Zhao, X. He, K. A. Hoadley, J. S. Parker, D. N. Hayes, and C. M. Perou, "Comparison of RNA-Seq by poly (A) capture, ribosomal RNA depletion, and DNA microarray for expression profiling," BMC Genomics, vol. 15, article 419, 2014. 

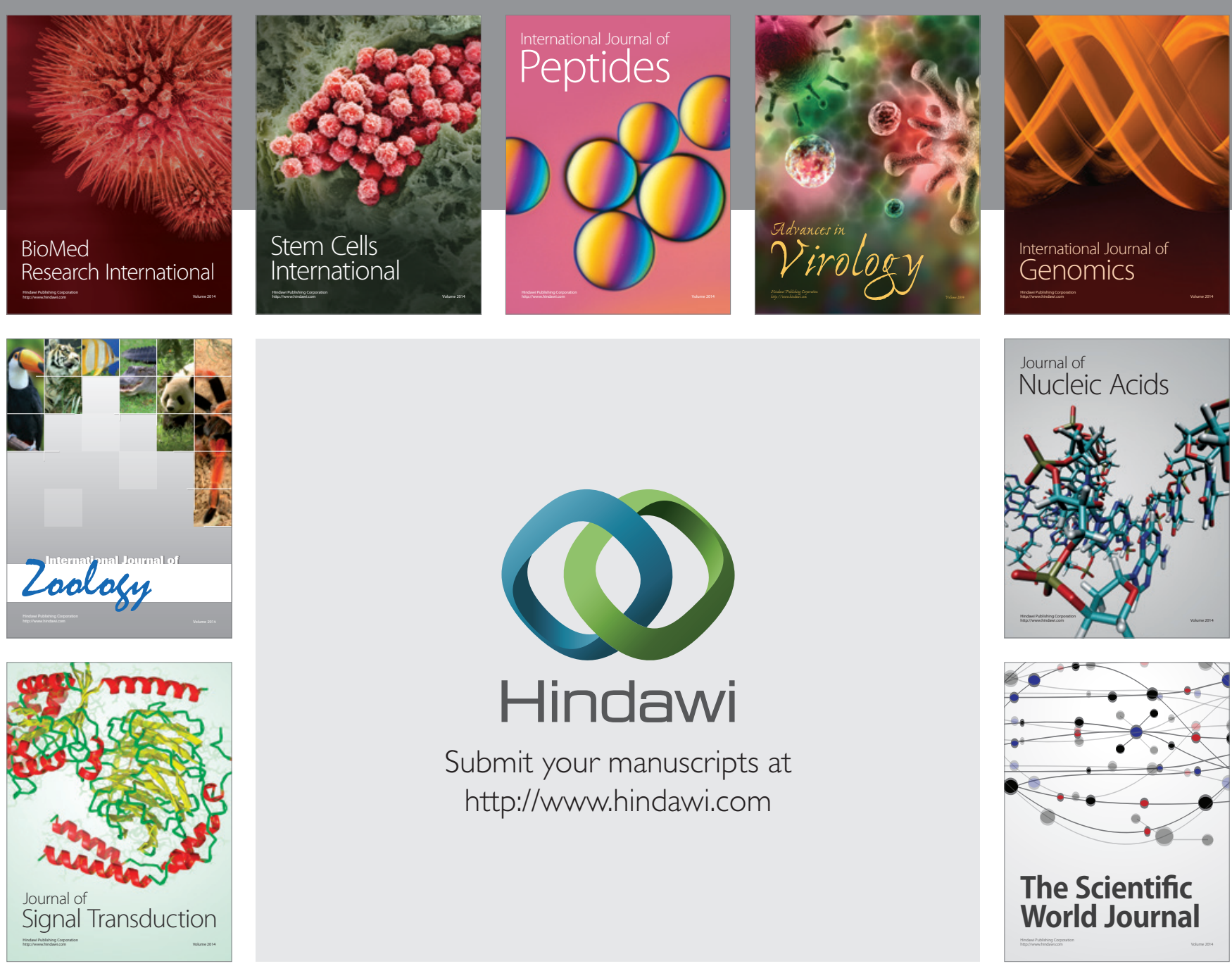

Submit your manuscripts at

http://www.hindawi.com
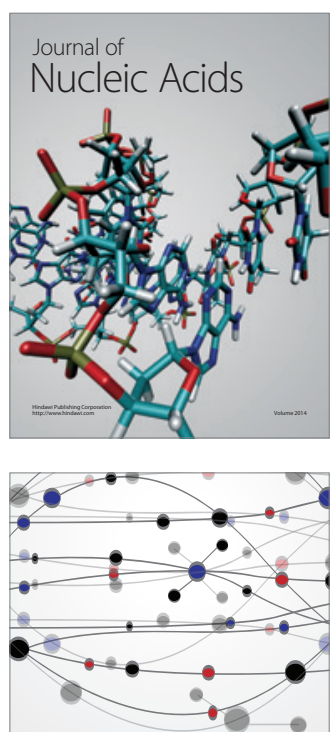

The Scientific World Journal
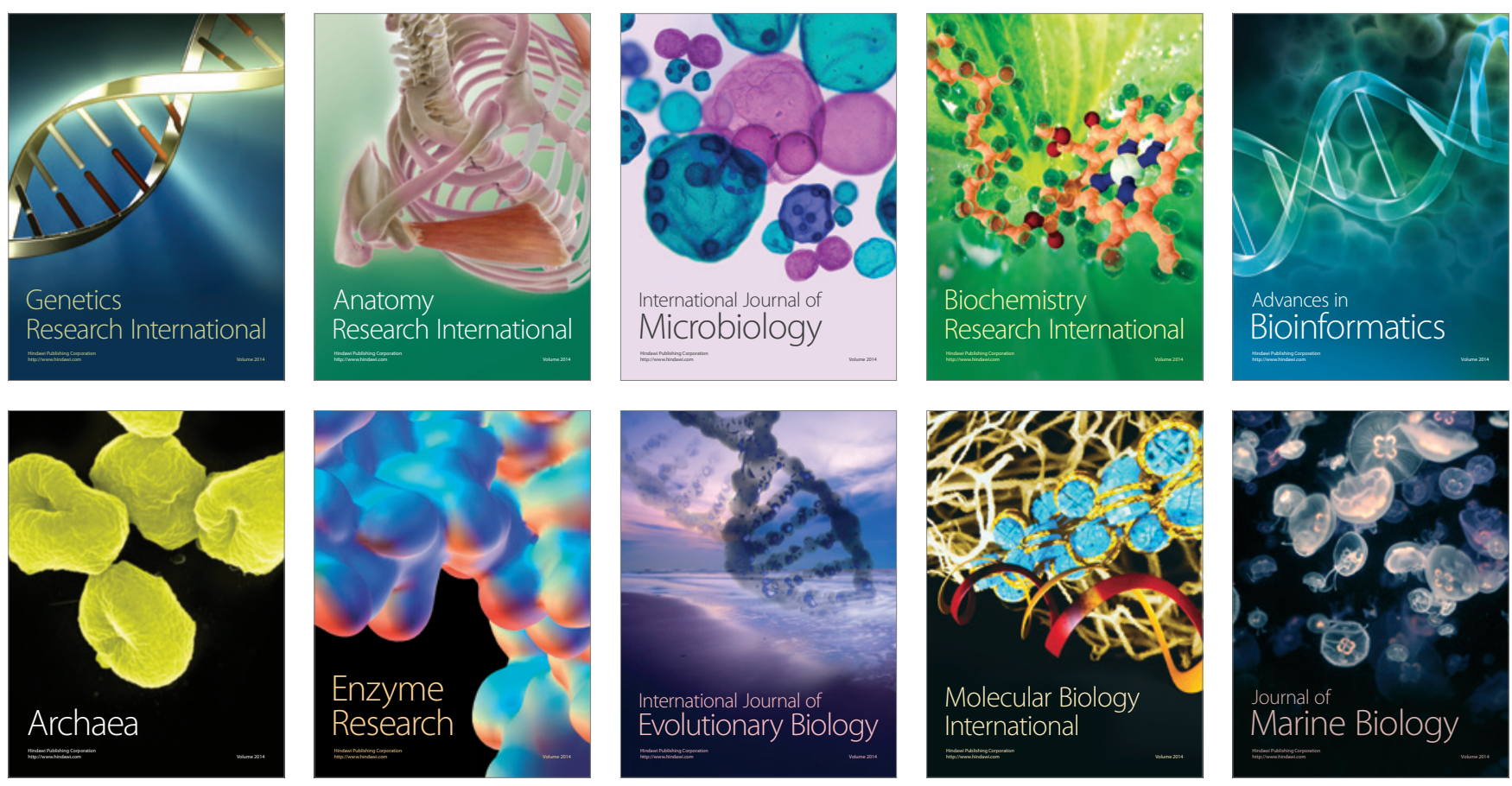